\title{
Change Point Detection in a State Space Framework Applied to Climate Change in Europe *
}

\author{
Magda Monteiro ${ }^{1,2[0000-0001-8585-4440]}$ and Marco Costa ${ }^{1,2[0000-0001-7686-2430]}$ \\ 1 Águeda School of Technology and Managment \\ University of Aveiro, Portugal \\ 2 CIDMA - Center for Research \& Development in Mathematics and Applications \\ University of Aveiro, Portugal \\ msvm@ua.pt \\ marco@ua.pt
}

\begin{abstract}
This work presents the statistical analysis of time series of monthly average temperatures in several European locations using a state space approach, where it is considered a model with a deterministic seasonal component and a stochastic trend. Temperature rise rates in Europe seem to have increased in the last decades when compared with longer periods, hence change point detection methods were applied to residuals state space models in order to identify these possible changes in the monthly temperature rise rates. In Northern Europe the change points were, almost all, identified in the late 1980s while in Central and Southeastern Europe was, for the majority of cities, in the 1990s and later.
\end{abstract}

Keywords: Air temperature. Climate change. state space models. Change point detection.

\section{Introduction}

Global warming and climate change are in the scientific agenda. The increase of droughts, floods, severe storms, and other weather catastrophes are putting vulnerable human and biological populations at risk. Nevertheless, global warming effects vary around the world, therefore this phenomenon must be monitored at a smaller scale, for instance at a European cities level. Statistical analysis of the evolution of climate variables can contribute to a more efficient identification and monitoring of patterns of change. In particular, the study of long temperature series is of particular interest in understanding climate dynamics at smaller scales, allowing efficient monitoring of environmental processes.

The application of state space models to long temperature time series in Lisbon, Coimbra, and Porto, in [6], allowed us to conclude that the latter city

\footnotetext{
* This work was partially supported the Center for Research and Development in Mathematics and Applications (CIDMA) through the Portuguese Foundation for Science and Technology (FCT - Fundação para a Ciência e a Tecnologia), references UIDB/04106/2020 and UIDP/04106/2020.
} 
has a very different growth rate per century than the others. In [5], the application of state space models to long series of air temperature, together with cluster procedures, let the identification of temperature growth rates patterns in several cities in Europe. This work allowed us to conclude that temperature rates have been increasing in the last decades when compared to longer periods. Following this work, we will analyze, in a state space framework, monthly temperature averages in these European cities aiming to identify the change points from which the pattern growth has changed and use them to accurately estimate growth rates for the forthcoming decades. A state space model comprises two equations, the state equation that translates the system dynamics, regulated by a latent process (the state), and the observation equation that establishes the link between the unobservable states and the observations. This class of models allows the description of both univariate and multivariate time series, stationary or not, that can present data irregularities such as interventions and missing data, reveling to be a flexible class. Moreover, this class has the advantage, in most cases, of detecting the temporal dynamics of the process more accurately when compared to other models. State space models have been successfully used in several research areas such as environment ([7],[4]), insurance ([3]), Finance ([22]) and animal ecology ([13]). Regarding structural breaks detection, there is a vast literature on the subject (see [2], [20] and [23], including the references therein). Much of the methodology was first developed for a sequence of independent and identically distributed (i.i.d.) random variables and a large part of the literature is devoted to CUSUM -type and likelihood ratio procedures based on distributional assumptions (usually Gaussian) to test for a change in the mean and or in variance. When no knowledge about the distributional form of the sequence under analysis is available, nonparametric change detection methods can be used in order to identify these changes. The works of [9] and [19] consider methods that have into account well established nonparametric tests for location and scale comparisons and [18] developed an R package ([15] ) with the implementation of several change points detection methods. The existence of serial dependence in many applications led to the development of methodologies to deal with change points in time series. Several approaches can be used in this context, for example one can apply test statistics developed for the independent setting to the underlying innovation process or one can try to quantify the effect of dependence on these test statistics depending on the model structure that is considered. There are several works on change point detection in climate or environmental settings. For instance, [16] makes a review of this topic in the climate context and [10] applied several change points procedures to Klementinum temperature. In line with this work, it is considered parametric and nonparametric statistical tests with the application of maximum type statistics to the the innovation processes predictions obtained by the Kalman filter algorithm for the temperature time series in Europe. 


\section{Data Description}

The Data is available at the Climate Data Online [12] and comprises the period between January 1900 and December 2017, making a total of 1416 observations. The location of the cities and data descriptive statistics can be found in Fig. 1 and Table 1 respectively. Table 1 presents the minimum, maximum and average temperature for all period and also the same statistics for period under the year 2000 and for the second millennium. The cutoff point was chosen in order to separate centuries. The global minimums are, with the exception of Ireland, in the first period and the global maximums were reached in the second period in two thirds of the series. For all the cities analyzed, the increase in average temperatures in recent years is quite evident.

Table 1. Characterization of time series data set.

\begin{tabular}{|c|c|c|c|c|c|c|c|}
\hline \multirow[t]{3}{*}{ city (country) } & \multirow{3}{*}{$\begin{array}{l}\min \max \\
1900 \\
\text { to } 2017\end{array}$} & \multirow{3}{*}{$\begin{array}{l}\min \max \\
1900 \\
\text { to } 1999\end{array}$} & \multicolumn{5}{|c|}{ min max average average average missing } \\
\hline & & & 2000 & 1900 & 1900 & 2000 & ralue \\
\hline & & & to 2017 & to 2017 to & 1999 to & 2017 & (\%) \\
\hline Berlin (Germany) & -10.723 .0 & -10.722 .5 & -4.823 .0 & 8.7 & 8.5 & 9.9 & 2.3 \\
\hline Bucharest (Romania) & -11.527 .5 & -11.527 .5 & -6.626 .5 & 10.1 & 9.8 & 11.1 & 2.6 \\
\hline Copenhagen (Denmark) & -6.721 .6 & -6.721 .6 & -3.121 .6 & 8.6 & 8.4 & 9.6 & 0.4 \\
\hline Dublin (Ireland) & 0.017 .1 & 0.417 .1 & 0.017 .0 & 9.2 & 9.2 & 9.6 & 2.2 \\
\hline Kiev (Ukraine) & -15.326 .0 & -15.326 .0 & -10.024 .7 & 8.1 & 7.9 & 9.4 & 0.9 \\
\hline Lisbon (Portugal) & 8.025 .1 & 8.025 .1 & 9.425 .1 & 16.9 & 16.8 & 17.4 & 3.4 \\
\hline Minsk (Belarus) & -16.822 .6 & -16.822 .0 & -11.122 .6 & 6.1 & 5.8 & 7.4 & 1.8 \\
\hline Nantes (France) & -1.223 .3 & -1.223 .2 & 2.523 .3 & 12.0 & 11.9 & 12.5 & 0.4 \\
\hline Prague (Czech Rep.) & -13.122 .0 & -13.121 .7 & -5.522 .0 & 7.9 & 7.6 & 9.1 & 8.2 \\
\hline S. Petersburg (Russia) & -18.324 .4 & -18.322 .4 & -12.124 .4 & 5.3 & 5.1 & 6.5 & 0.0 \\
\hline Talin (Estonia) & -15.521 .5 & -15.521 .2 & -1121.5 & 5.3 & 5.1 & 6.5 & 5.4 \\
\hline Vienna (Austria) & -9.524 .4 & -9.524 .4 & -3.424 .1 & 10.1 & 9.9 & 11.3 & 0.2 \\
\hline Vilnius (Lithuania) & -17.121 .8 & -17.120 .4 & -10.421 .8 & 5.8 & 5.6 & 7.3 & 2.6 \\
\hline Zagreb (Croatia) & -7.025 .9 & -7.025 .9 & -2.525 .7 & 11.8 & 11.6 & 13.0 & 1.4 \\
\hline Zurich (Switzerland) & -8.722 .4 & -8.721 .8 & -3.622 .4 & 8.7 & 8.5 & 9.8 & 0.0 \\
\hline
\end{tabular}

\section{Model and Change Point Methods Descriptions}

\subsection{Model}

Assuming that there are no change-points, the model is defined, for all time sequence, by

$$
\begin{aligned}
Y_{t} & =\tau_{t}+\sum_{i=1}^{12} \beta_{i ; 0} S_{t, i}+e_{1, t} \\
\tau_{t} & =\mu_{0}+\phi_{0}\left(\tau_{t-1}-\mu_{0}\right)+e_{2, t}
\end{aligned}
$$




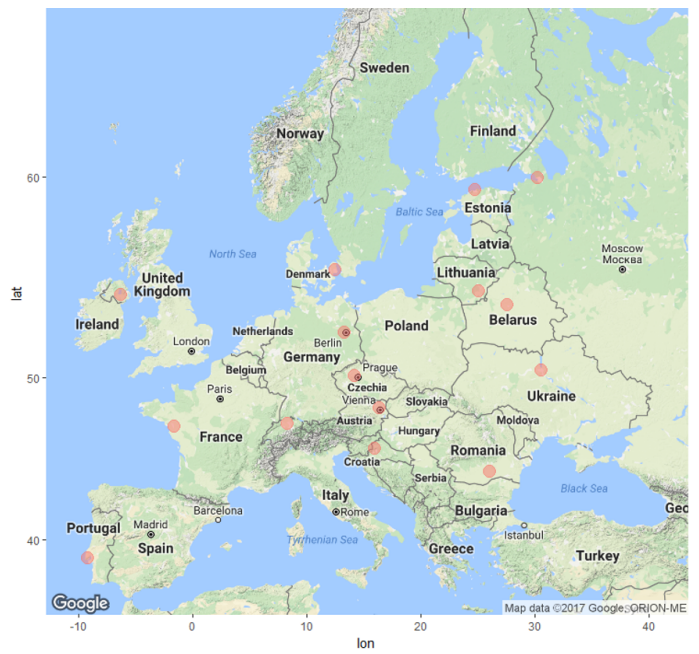

Fig. 1. Station locations (Adapted from google earth)

where $Y_{t}$ represents the monthly average air temperature in the month $t$, with $t=1, \ldots, n$, and $\beta_{i ; 0}$ with $i=1,2, \ldots, 12$ is the seasonal coefficient associated to the month $t=i+12 k$, for some $k=0,1,2, \ldots, S_{t, i}$ is an indicative function defined such that

$$
S_{t, i}=1=\left\{\begin{array}{l}
1 \text { if } t=i+12 k, \text { for some } k=0,1,2, \ldots \\
0 \quad \text { otherwise }
\end{array}\right.
$$

$\left\{\tau_{t}\right\}$ is the trend component that follows a stationary autoregressive process with mean $\mu_{0}$ with autoregressive parameter $\left|\phi_{0}\right|<1$; the error $e_{i, t}$, with $i=1,2$, follows a white noise process $\left(E\left(e_{i, t}\right)=0, \operatorname{var}\left(e_{i, t}\right)=\sigma_{i ; 0}^{2}\right.$ and $E\left(e_{i, t} e_{i, r}\right)=0$, for $t \neq r)$, and are uncorrelated errors, that is $E\left(e_{1, t} e_{2, r}\right)=0, \forall t, r$.

The error $e_{1, t}$ is called the observation error and it can be seen as a measure error, whereas the error $e_{2, t}$ is called the state error and translates the randomness of the trend component. Note that state $\tau_{t}$ is an unobservable process and its predictions must be obtained. Kalman filter algorithms provides optimal unbiased estimators for the state $\tau_{t}$; $\widehat{\tau}_{t \mid t-1}$ for one-step-ahead prediction, $\widehat{\tau}_{t \mid t}$ for Kalman filter update and $\widehat{\tau}_{t \mid n}$ for Kalman smoother predictions ([8]).

It is noted that no assumptions are made to distributional form of both errors, although in many situations the Gaussian distribution is considered.

\subsection{Change Point Detection}

In order to discover an abrupt change in the behavior of the temperature rise rates, a basic statistical tests applying maximum type statistics to detect a change in location are performed. If there are no structural changes in the temperature trend, it is expected that $\tau_{t}$ has no changes over time in its mean. If no 
change point exists, the one-step-ahead forecast residuals are independent and identically. If a change point exists at some time $t^{*}$, the one-step-ahead forecast innovations can be used to detect and estimate it [19], and it is assumed that after that time point the parameters of model $1-2$ change to $\beta_{i ; 1}, i=1, \cdots, 12$, $\mu_{1}, \phi_{1}$ and $\sigma_{j ; 1}^{2}, j=1,2$. We used Maximum type tests for both parametric and nonparametric techniques.

First, consider that it is intended to test if the change point occurs at the known time point $k$. The statistical hypotheses are

$$
\begin{aligned}
& H_{0}: X_{t} \sim F_{0}\left(x ; \theta_{0}\right) \text { for } \forall t=1 \cdots, n \\
& \text { vs } \\
& H_{1}: X_{t} \sim F_{0}\left(x ; \theta_{0}\right) \text { for } t \leq k \text { and } X_{t} \sim F_{1}\left(x ; \theta_{1}\right), \text { for } t>k .
\end{aligned}
$$

Under the non existence of change points hypothesis, it can be applied a, parametric or a nonparametric, two-sample comparison test. If the statistic test exceeds some appropriately chosen threshold then the null hypothesis that the two samples have identical distributions is rejected, and we conclude that a change point has occurred immediately after $k$ time. Since it is not known in advance where the change point occurs, the $k$ time is unknown and the statistic test is evaluated at every value $1<k<n$, and the maximum value is used.

Maximum type $\mathbf{T}$ test Under normality assumption, with no changes in $\sigma^{2}$ which is assumed to be unknown, the test statistic $T(n)$ is the maximum of the absolute values of two sample $t$-test statistics

$$
T(n)=\max _{1 \leq k<n}\left|T_{k}\right|=\max _{1 \leq k<n} \sqrt{\frac{(n-k) k}{n}} \frac{\left|\bar{X}_{k}-\bar{X}_{k}^{*}\right|}{s_{k}}
$$

where

$$
\bar{X}_{k}=\frac{1}{k} \sum_{i=1}^{k} X_{i}, \bar{X}_{k}^{*}=\frac{1}{n-k} \sum_{i=k+1}^{n} X_{i}
$$

and

$$
s_{k}=\sqrt{\frac{1}{n-2}\left[\sum_{i=1}^{k}\left(X_{i}-\bar{X}_{k}\right)^{2}+\sum_{i=k+1}^{n}\left(X_{i}-\bar{X}_{k}^{*}\right)^{2}\right]} .
$$

The null hypothesis can be rejected if the statistic $T(n)$ is greater than the critical value. The exact distribution of $T(n)$, for independent variables, is very complex and [21] was able to calculate the true critical values only for the number of observations $n$ less than 10. Alternatively, approximate critical values can be computed by other methods as the Bonferroni inequality, simulation or the asymptotic distribution.

When random variables $X_{1}, X_{2}, \ldots, X_{n}$ are not independent but form an ARMA process then the asymptotic critical values of the test statistics considering independence have to be multiplied by $\sqrt{2 \pi f(0) / \gamma}$, where $\gamma=\operatorname{var}\left(X_{t}\right)$ 
and $f(\cdot)$ denotes the spectral density function of the corresponding ARMA process ( [1]). Especially for an $\operatorname{AR}(1)$ sequence, the critical values should be multiplied by $\left[(1+\phi)(1-\phi)^{-1}\right]^{1 / 2}$ where $\phi$ is the first autoregressive coefficient, [11].

Maximum type Mann-Whitney test In the nonparametric setting, at each $k$ point, $1<k<n$, the two-sample Mann-Whitney test statistic $D_{k ; n}$ can be computed. The variance of $D_{k ; n}$ depends on the value $k$, so the test statistic is obtained through the maximization of the standardized $D_{k, n}$ to have mean 0 and variance 1 (see [18]),

$$
D_{n}=\max _{k=2, \cdots, n-1} \widetilde{D}_{k, n}=\left|\frac{D_{k, n}-\mu_{D_{k, n}}}{\sigma_{D_{k, n}}}\right| .
$$

The null hypothesis of no change is then rejected if $D_{n}>h_{n}$ for some appropriately chosen threshold $h_{n}$. This distribution does not have an analytic finite-sample form and for the case of the Mann-Whitney statistics the asymptotic distribution of $D_{n}$ can be written ([14]). However, these asymptotic distributions may not be accurate when considering finite length sequences, and so numerical simulation may be required in order to estimate the distribution $([17,18])$. The best estimate of the change point location will be immediately following the value of $\mathrm{k}$ which maximized $\widetilde{D}_{n, k}$.

\section{Results}

The parameters of model 1-2 were estimated in a classical decomposition approach combining the least squares estimation of the seasonal parameters $\beta_{0 ; i}$, with $i=1, \ldots, 12$, with a distribution-free estimators developed to state space models (see [7]) in order to estimate the remaining parameters. The results of the estimation procedure are presented in Table 2 .

All $\phi$ estimates verifies the stationary condition of the AR(1) process and the smallest estimate of the mean of the stochastic trend level is 0.383 in Dublin (Ireland) whereas the largest is 1.132 in Vilnius (Lithuania). The residuals analysis showed that, for the majority of cities, there is no serial correlation up to lag 10 when considering a significance of $1 \%$ (see Fig. 2). The exception is the city of Lisbon which presents no serial correlation only up to lag 3.

Although no assumption was made to the distribution of the errors in the model under study, the normality was tested through the application of JarqueBera test to standard residuals which lead to the rejection of this assumption in the majority of the cities. This situation is not unusual, since the length of the series is quite long, and in this situations tests tend to reject quite often.

This is an ongoing work and we only used the at most one change point approach. For each city we have considered three situations: the maximum-type test using Mann-Whitney tests and also $\mathrm{T}$ test applied to the residuals; the application of the maximum-type $\mathrm{T}$ test applied to smoother predictions of the 
Table 2. Parameter estimates of model 1-2

\begin{tabular}{|c|c|c|c|c|c|c|c|c|c|c|c|c|c|c|c|}
\hline \multirow[b]{2}{*}{ city } & \multicolumn{11}{|c|}{$\widehat{\beta_{0}}$} & \multirow[b]{2}{*}{$\widehat{\mu}_{0}$} & \multirow[b]{2}{*}{$\widehat{\phi}_{0}$} & \multirow[b]{2}{*}{$\widehat{\sigma}_{0 ; e_{1}}^{2}$} & $\widehat{\sigma}_{0 ; e_{2}}^{2}$ \\
\hline & Jan. & $\mathrm{Fe}$ & Ma & $\mathrm{Ap}$ & . May & Jun. Jul. & Aug & Sep. & Oct. & Nov. & Dec. & & & & $\widehat{\sigma}_{0 ; Y_{t}}^{2}$ \\
\hline Berlin & -1.2 & -0.4 & 3.0 & 7.6 & 12.7 & 15.817 .5 & 16.6 & 12.9 & 8.1 & 3.2 & 0.1 & 0.709 & 0.576 & 1.937 & 1.3193 .912 \\
\hline Bucharest & -3.9 & -1.7 & 3.6 & 9.9 & 15.3 & 19.121 .3 & 20.7 & 16.0 & 9.8 & 3.8 & -1.4 & 0.611 & 0.537 & 1.989 & 1.6534 .311 \\
\hline Copenhagen & 0.0 & -0.2 & 1.9 & 6.1 & 11.2 & 14.916 .9 & 16.4 & 13.0 & 8.7 & 4.5 & 1.7 & 0.655 & 0.711 & 1.128 & 8362.815 \\
\hline Dublin & 4.4 & 4.5 & 5.6 & 7.3 & 9.9 & 12.714 .5 & 14.2 & 12.3 & 9.6 & 6.3 & 5.0 & 0.383 & 0.52 & 0 & 1.369 \\
\hline Kiev & -5.5 & -4.7 & 0.2 & 8.0 & 14.6 & 17.919 .6 & 18.7 & 13.7 & 7.4 & 1.4 & -3.1 & 0.732 & 0.583 & 2.735 & 1.8935 .601 \\
\hline Lisbor & 10.9 & 911.6 & 13.4 & 14.9 & 17.0 & 19.921 .8 & 22.3 & 20.9 & 17.9 & 14.0 & 11 & 0 & - & 0 . & 299 \\
\hline Minsk & -7.1 & -6.4 & -2.1 & 5.3 & 11.8 & 15.417 .0 & 16.0 & 11.1 & 5.2 & -0.3 & -4.6 & 0.95 & 0.679 & 3.377 & 95.788 \\
\hline Nantes & 5.2 & 5.7 & 7.9 & 10.2 & 13.6 & 16.618 .6 & 18.3 & 16.0 & 12.2 & 8.0 & 5.8 & 0. & 0.439 & 1. & 352.357 \\
\hline Prague & -2.8 & -1.8 & 2.0 & 6.7 & 11.9 & $15,116.9$ & 16.2 & 12.3 & 7.1 & 1.9 & -1.4 & 0.797 & 0.701 & 2.506 & 3.831 \\
\hline S. Petersburg & -7.5 & -7.5 & -3.4 & 3.3 & 9.9 & 14.817 .7 & 15.9 & 10.7 & 4.8 & -0.6 & -4.9 & 0.8 & 0.607 & 3.232 & 2.1006 .560 \\
\hline Talin & -5.5 & -6.0 & -2.8 & 2.7 & 8.6 & 13.216 .0 & 15.0 & 10.5 & 5.3 & 0.5 & -3.0 & 0.80 & 0.6 & 2.1 & 1.8445 .360 \\
\hline Vienna & -1.0 & 0.5 & 4.6 & 9.5 & 14.3 & 17.519 .4 & 18.7 & 14.7 & 9.4 & 4.3 & 0.6 & 0.710 & 0.559 & 2.043 & 1.0863 .624 \\
\hline Vilnius & -6.7 & -5.9 & -2.3 & 4.5 & 11.0 & 14.316 .2 & 15.1 & 10.4 & 4.9 & -0.4 & -4.3 & 1.132 & 0.821 & 3.487 & 0.7825 .889 \\
\hline Zagreb & 0.3 & 2.4 & 6.9 & 11.5 & 16.0 & 19.421 .4 & 20.8 & 16.7 & 11.4 & 6.2 & 1.9 & 0.572 & 0.60 & 2.258 & 1.0443 .907 \\
\hline Zurich & -1.2 & 0.0 & 3.8 & 7.5 & 11.9 & 15.116 .9 & 16.3 & 13.1 & 8.2 & 3.1 & -0.1 & 0.763 & 0.653 & 2.302 & 0.5913 .331 \\
\hline
\end{tabular}

\section{Ljung-Box test for Residuals up to lag 10}

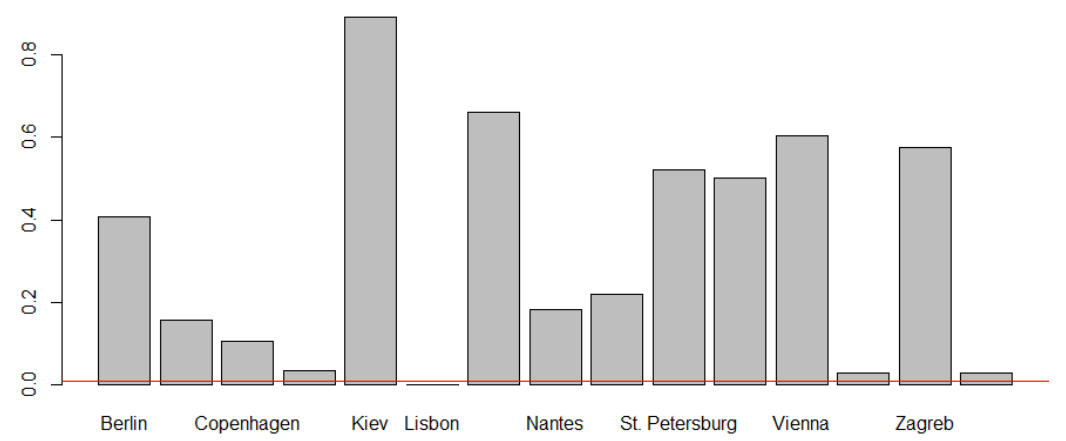

Fig. 2. Ljung-Box p-values residuals tests. $\alpha=1 \%$ in red. 
latent process $\tau_{t}$ which represents the trend of the temperature. Since $\tau_{t}$ has an autoregressive structure of order 1 , it was necessary to adjust, for each series, the critical value of the test using the estimates of the autoregressive parameters. Results of the change point detection are presented in Table 3.

Table 3. Change point time for each city;

\begin{tabular}{lccc}
\hline & M-W test & T-test & t-test (smoother) \\
change point change point & $\begin{array}{c}\text { change point } \\
\text { month/year }\end{array}$ & month/year & month/year \\
\hline Berlin (Germany) & $8 / 1987$ & $8 / 1987$ & $9 / 1987$ \\
Bucharest (Romania) & $9 / 2006$ & $12 / 2007$ & $9 / 2006$ \\
Copenhagen (Denmark) & $8 / 1987$ & $8 / 1987$ & $9 / 1987$ \\
Dublin (Ireland) & $9 / 1987$ & $9 / 1988$ & $10 / 1988$ \\
Kiev (Ukraine) & $12 / 1999$ & $11 / 1988$ & $12 / 1989$ \\
Lisbon (Portugal) & $1 / 1945$ & $6 / 1978$ & $7 / 1978$ \\
Minsk (Belarus) & $11 / 1988$ & $11 / 1987$ & $11 / 1987$ \\
Nantes (France) & $7 / 1981$ & $8 / 2017$ & $7 / 1987$ \\
Prague (Czech Rep.) & $11 / 1997$ & $8 / 1987$ & $8 / 1987$ \\
S. Petersburg (Russia) & $12 / 1987$ & $12 / 1988$ & $12 / 1988$ \\
Talin (Estonia) & $9 / 1987$ & $9 / 1987$ & $12 / 1988$ \\
Vienna (Austria) & $8 / 1987$ & $9 / 1987$ & $8 / 1987$ \\
Vilnius (Lithuania) & $6 / 1982$ & $9 / 1987$ & $11 / 1987$ \\
Zagreb (Croatia) & $12 / 1991$ & $12 / 1992$ & $12 / 1992$ \\
Zurich (Switzerland) & $7 / 1997$ & $6 / 1987$ & $8 / 1987$ \\
\hline
\end{tabular}

There are several cities whose change points obtained by the three methods are very similar. In most of these cities the change points are identified in the late eighties or in the early nineties of the last century, with the exception of Bucharest which was identified in 2006. Although normality assumption was rejected, the results based on the T-tests do not differ substantially from the results obtained in the nonparametric case.

We highlight Kiev, Prague and Zurich as cities where the nonparametric method identifies a change point about ten years later than the other two methods. In Lisbon case the nonparametric method identifies a change point about thirty years early than the other methods. Analysing the values of the statistical tests involved there is two local maximums indicating the possibility of the existence of more than one change point (Fig. 3). Note that in Lisbon the residuals show significant correlation after lag 3 , so in this case the tests validity are compromised and is necessary further model investigation in order to have non correlated residuals.

Table 3 presents discrepant values in Nantes. The nonparametric method points for a change point in July 1981 and the $\mathrm{T}$ test applied to smoother predictions points for a change point six years later. The $\mathrm{T}$ test applied to the residuals presents a change point almost at the end of the period under analysis. The first difference can be explained by the fact that between 1981 and 1987 

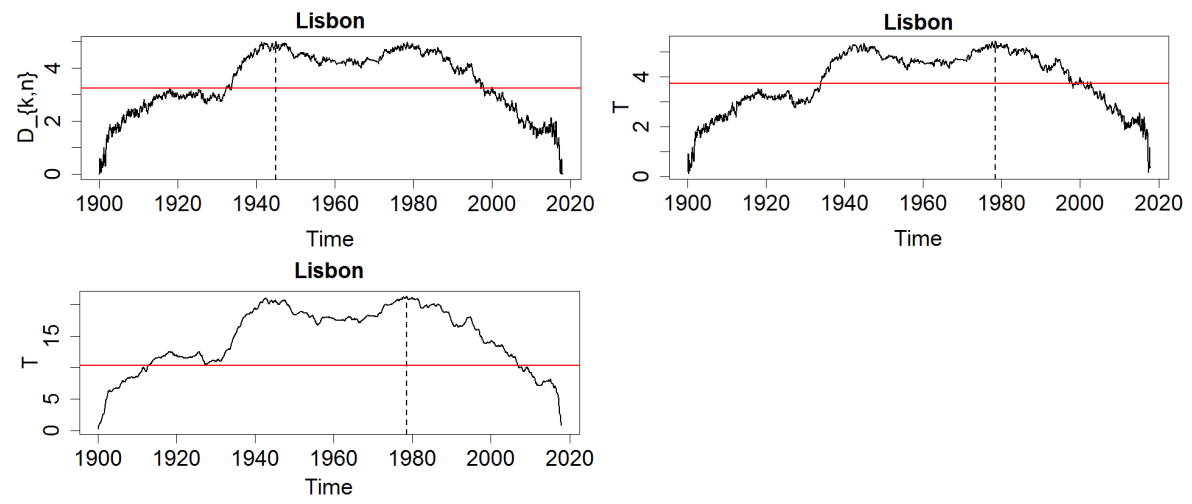

Fig. 3. Values of $\widetilde{D}_{k, n}$ for residuals,$T_{k}$ for residuals and $T_{k}$ for smoother predictions in Lisbon.

there are two local maximums in the test statistics. In the nonparametric case the global maximum occur in july 1981 but the difference for the other value is rather small, 0.08 (see Fig. 4). In july 2017 there is a spike in all test statistics, nevertheless only in the $\mathrm{T}$ test applied to the residuals this value exceeds all other values. Note that in the test statistic, $T_{k}$, the mean of the second segment is calculated with only 5 observations. We highlight the fact that the residuals from July 2017 are very high compared to the other values of the series which may justify the discrepancy with the other methods.
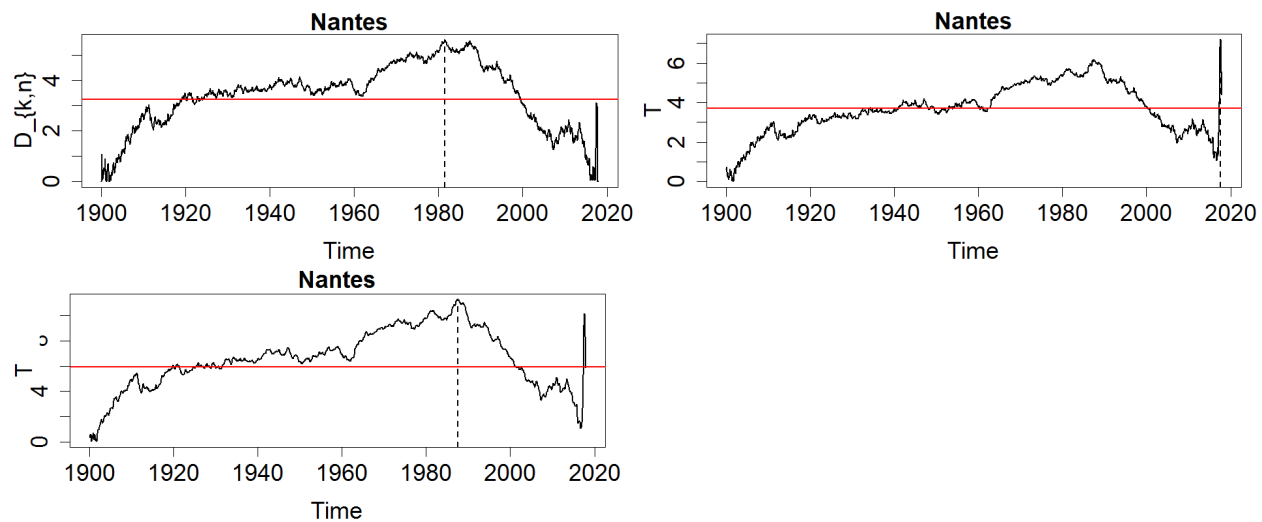

Fig. 4. Values of $\widetilde{D}_{k, n}$ for residuals, $T_{k}$ for residuals and $T_{k}$ for smoother predictions in Nantes.

In order to analyze the existence of a geographical pattern in the detection of change points, Fig. 5 presents, for each city and the nonparametric method, the time (in terms of early or late decade) identified as a change point. In Northern 


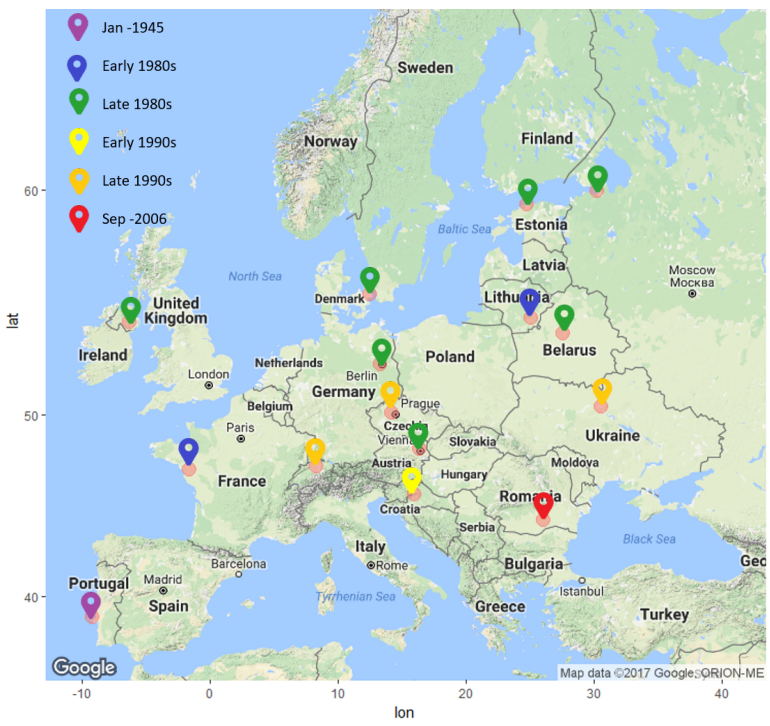

Fig. 5. Change point identification, for each city, using the nonparametric approach (adapted from google earth)

Table 4. Residuals mean before and after change point time defined by $\mathrm{M}-\mathrm{W}$ test

\begin{tabular}{lccc}
\hline city (country) & $\begin{array}{c}\text { mean residuals } \\
\text { before change point after }\end{array}$ & $\begin{array}{c}\text { mean residuals } \\
\text { change point }\end{array}$ & $\begin{array}{c}\text { Mean } \\
\text { Difference }\end{array}$ \\
\hline Berlin (Germany) & -0.172 & 0.573 & 0.745 \\
Bucharest (Romania) & -0.075 & 0.93 & 1.005 \\
Copenhagen (Denmark) & -0.121 & 0.371 & 0.492 \\
Dublin (Ireland) & -0.092 & 0.305 & 0.397 \\
Kiev (Ukraine) & -0.131 & 0.756 & 0.887 \\
Lisbon (Portugal) & -0.188 & 0.126 & 0.314 \\
Minsk (Belarus) & -0.215 & 0.635 & 0.850 \\
Nantes (France) & -0.15 & 0.353 & 0.503 \\
Prague (Czech Rep.) & -0.144 & 0.693 & 0.837 \\
S. Petersburg (Russia) & -0.199 & 0.598 & 0.797 \\
Talin (Estonia) & -0.175 & 0.475 & 0.650 \\
Vienna (Austria) & -0.173 & 0.54 & 0.713 \\
Vilnius (Lithuania) & -0.212 & 0.491 & 0.703 \\
Zagreb (Croatia) & -0.157 & 0.609 & 0.766 \\
Zurich (Switzerland) & -0.133 & 0.685 & 0.818 \\
\hline
\end{tabular}

Europe the change points were, with the exception of Vilnius, identified in the late 1980s while in Central and Southeastern Europe, with Vienna one exception, this identification was in the 1990s and later. As mentioned before Lisbon case, in southwestern Europe, presents a change point much earlier than the other cities. Table 4 presents the residuals means before and after the detection of the 
change points using the nonparametric approach with the $\mathrm{M}-\mathrm{W}$ tests. One can see, together with Table 3 and Fig. 5, that the biggest differences occur almost always for later detections and that the smaller changes occur in western Europe.

\section{Conclusions}

By applying methodologies to detect at most one change point in the residuals of the state space models applied to monthly average temperatures of European cities, it was possible to conclude that they exist and are different, in time and magnitude, for these different cities of Europe.

From this detection, and for each city, it is important to use it in the estimation of the parameters before and after the change point in order to assess the existence or not of more change points and, in a final stage, to use the most appropriate model to predict the temperature rise rates in European cities. This is a topic of future research.

\section{References}

1. Antoch, J., Huškova, M., Prášková, Z.: Effect of dependence on statistics for determination of change. Journal of Statistical Planning and Inference 60(2), 291-310 (1997). https://doi.org/https://doi.org/10.1016/S0378-3758(96)00138-3, https://www.sciencedirect.com/science/article/pii/S0378375896001383

2. Aue, A., Horváth, L.: Structural breaks in time series. Journal of Time Series Analysis 34(1), 1-16 (2013). https://doi.org/https://doi.org/10.1111/j.14679892.2012.00819.x, https://onlinelibrary.wiley.com/doi/abs/10.1111/j.14679892.2012.00819.x

3. Chukhrova, N., Johannssen, A.: State space models and the kalman-filter in stochastic claims reserving: Forecasting, filtering and smoothing. Risks 5(2) (2017). https://doi.org/10.3390/risks5020030, https://www.mdpi.com/2227-9091/5/2/30

4. Costa, M., Gon,calves, A.: Clustering and forecasting of dissolved oxygen concentration on a river basin. Stoch Environ Res Risk Assess 25, 151-163 (2011). https://doi.org/10.1007/s00477-010-0429-5

5. Costa, M., Monteiro, M.: Statistical modeling of an air temperature time series of european cities. In: Advances in Environmental Research, pp. 213-236. Nova Science (2017)

6. Costa, M., Monteiro, M.: A periodic mixed linear state space model to monthly long-term temperature data. Environmetrics pp. 1-20 (2018). https://doi.org/10.1002/env.2550

7. Costa, M., Alpuim, T.: Parameter estimation of state space models for univariate observations. Journal of Statistical Planning and Inference 140(7), 1889-1902 (2010). https://doi.org/https://doi.org/10.1016/j.jspi.2010.01.036, https://www.sciencedirect.com/science/article/pii/S0378375810000479

8. Harvey, A.: Forecasting structural time series models and the Kalman filter. Cambridge University Press (2006)

9. Hawkins, D.M., Deng, Q.: A nonparametric change-point control chart. Journal of Quality Technology 42(2), 165173 (2010). https://doi.org/10.1080/00224065.2010.11917814, https://doi.org/10.1080/00224065.2010.11917814 
10. Jarušková, D., Antoch, J.: Changepoint analysis of klementinum temperature series. Environmetrics 31(1), e2570 (2020). https://doi.org/https://doi.org/10.1002/env.2570, https://onlinelibrary.wiley.com/doi/abs/10.1002/env.2570, e2570 env.2570

11. Jarušková, D.: Some problems with application of change-point detection methods to environmental data. Environmetrics 8(5), 469-483 (1997). https://doi.org/https://doi.org/10.1002/(SICI)1099095X(199709/10)8:5;469::AID-ENV265¿3.0.CO;2-J,

12. National Centers for Environmental Information: Climate Data Online. https://www.ncdc.noaa.gov/cdo-web, last checked: 2019-01-10

13. Patterson, T.A., Thomas, L., Wilcox, C., Ovaskainen, O., Matthiopoulos, J.: State-space models of individual animal movement. Trends in Ecology Evolution 23(2), 87-94 (2008). https://doi.org/https://doi.org/10.1016/j.tree.2007.10.009, https://www.sciencedirect.com/science/article/pii/S0169534707003588

14. Pettitt, A.N.: A non-parametric approach to the change-point problem. Journal of the Royal Statistical Society. Series C (Applied Statistics) 28(2), 126-135 (1979), http://www.jstor.org/stable/2346729

15. R Core Team: R: A Language and Environment for Statistical Computing. R Foundation for Statistical Computing, Vienna, Austria (2013), http://www.Rproject.org/

16. Reeves, J., Chen, J., Wang, X.L., Lund, R., Lu, Q.Q.: A review and comparison of changepoint detection techniques for climate data. Journal of Applied Meteorology and Climatology 46(6), 900 - 915 (2007). https://doi.org/10.1175/JAM2493.1, https://journals.ametsoc.org/view/journals/apme/46/6/jam2493.1.xml

17. Ross, G.J.: cpm: Sequential and Batch Change Detection Using Parametric and Nonparametric Method (2015), r package version 2.2

18. Ross, G.J.: Parametric and nonparametric sequential change detection in R: The cpm package. Journal of Statistical Software 66(3), 1-20 (2015), http://www.jstatsoft.org/v66/i03/

19. Ross, G.J., Tasoulis, D.K., Adams, N.M.: Nonparametric monitoring of data streams for changes in location and scale. Technometrics 53(4), 379-389 (2011). https://doi.org/10.1198/TECH.2011.10069, https://doi.org/10.1198/TECH.2011.10069

20. Shao, X., Zhang, X.: Testing for change points in time series. Journal of the American Statistical Association 105(491), 1228-1240 (2010). https://doi.org/10.1198/jasa.2010.tm10103, https://doi.org/10.1198/jasa.2010.tm10103

21. Worsley, K.J.: On the likelihood ratio test for a shift in location of normal populations. Journal of the American Statistical Association 74(366), 365-367 (1979), http://www.jstor.org/stable/2286336

22. Zandonade, E., Morettin, P.A.: Wavelets in state space models. Applied Stochastic Models in Business and Industry 19(3), 199-219 (July 2003). https://doi.org/10.1002/asmb.496

23. Zou, C., Yin, G., Feng, L., Wang, Z.: Nonparametric maximum likelihood approach to multiple change-point problems. The Annals of Statistics 42(3), 970 - 1002 (2014). https://doi.org/10.1214/14-AOS1210, https://doi.org/10.1214/14AOS1210 